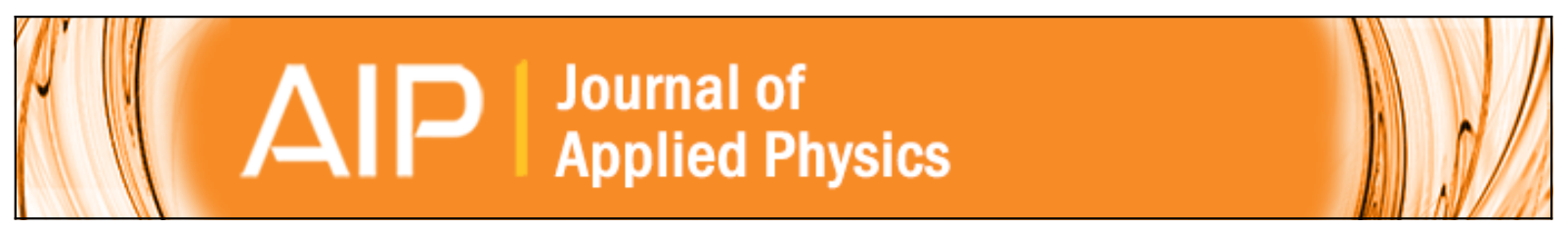

\title{
Phase transition and enhanced magneto-dielectric response in BiFeO3-DyMnO3 multiferroics
}

Satya N. Tripathy, Dhiren K. Pradhan, Karuna K. Mishra, Shrabanee Sen, Ratnakar Palai, Marian Paulch, James F. Scott, Ram S. Katiyar, and Dillip K. Pradhan

Citation: Journal of Applied Physics 117, 144103 (2015); doi: 10.1063/1.4916927

View online: http://dx.doi.org/10.1063/1.4916927

View Table of Contents: http://scitation.aip.org/content/aip/journal/jap/117/14?ver=pdfcov

Published by the AIP Publishing

\section{Articles you may be interested in}

$\mathrm{Mn}$ doping instigated multiferroicity and magneto-dielectric coupling in $\mathrm{KNbO} 3$

J. Appl. Phys. 118, 234105 (2015); 10.1063/1.4938118

Impedance and AC conductivity study of nano crystalline, fine grained multiferroic bismuth ferrite (BiFeO3), synthesized by microwave sintering

AlP Advances 5, 097164 (2015); 10.1063/1.4931818

Effect of Dy-substitution on the structural, vibrational, and multiferroic properties of $\mathrm{BiFeO} 3$ nanoparticles

J. Appl. Phys. 115, 214109 (2014); 10.1063/1.4881529

Room temperature multiferroic properties and magnetoelectric coupling in Sm and Ni substituted $\mathrm{Bi} 4-\mathrm{x} \mathrm{Sm} x$ $\mathrm{Ti} 3-\mathrm{x}$ Ni $x$ 012 $\pm \delta(x=0,0.02,0.05,0.07)$ ceramics

J. Appl. Phys. 115, 204909 (2014); 10.1063/1.4880159

Phase transition and magneto-electric coupling of $\mathrm{BiFeO} 3-\mathrm{YMnO} 3$ multiferroic nanoceramics

J. Appl. Phys. 114, 144104 (2013); 10.1063/1.4824061

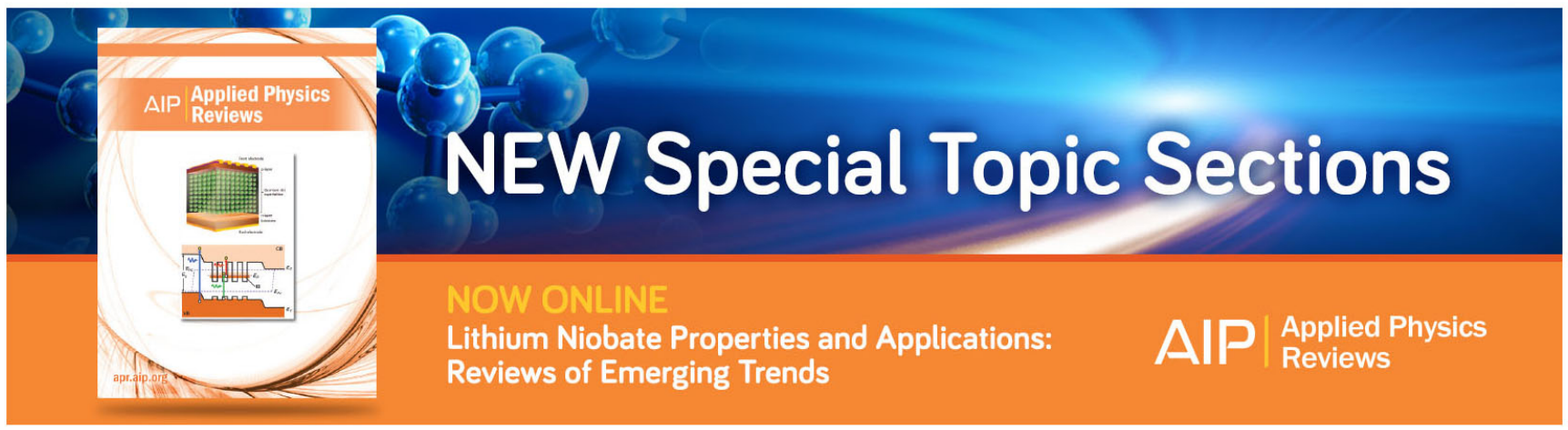




\title{
Phase transition and enhanced magneto-dielectric response in $\mathrm{BiFeO}_{3}-\mathrm{DyMnO}_{3}$ multiferroics
}

\author{
Satya N. Tripathy, ${ }^{1, a)}$ Dhiren K. Pradhan, ${ }^{2}$ Karuna K. Mishra, ${ }^{3}$ Shrabanee Sen, ${ }^{4}$ \\ Ratnakar Palai, ${ }^{2}$ Marian Paulch, ${ }^{5}$ James F. Scott, ${ }^{6}$ Ram S. Katiyar, ${ }^{2}$ and Dillip K. Pradhan ${ }^{1, b)}$ \\ ${ }^{1}$ Department of Physics and Astronomy, National Institute of Technology, Rourkela 769008, India \\ ${ }^{2}$ Department of Physics and Institute for Functional Nanomaterials, University of Puerto Rico, San Juan, \\ Puerto Rico 00936, USA \\ ${ }^{3}$ Condensed Matter Physics Division, Indira Gandhi Centre for Atomic Research, Kalpakkam 603102, India \\ ${ }^{4}$ Sensor and Actuator Division, Central Glass and Ceramics Research Institute, Kolkata 700032, India \\ ${ }^{5}$ Institute of Physics, University of Silesia, Uniwersytecka 4, 40-007 Katowice, Poland \\ ${ }^{6}$ Department of Physics, Cavendish Laboratory, University of Cambridge, Cambridge CB3 OHE, \\ United Kingdom
}

(Received 5 January 2015; accepted 23 March 2015; published online 9 April 2015)

\begin{abstract}
We report systematic studies on crystal structure and magneto-dielectric properties of $(1-x)$ $\mathrm{BiFeO}_{3}-x \mathrm{DyMnO}_{3}(0.0 \leq x \leq 0.2)$ nanoceramics synthesized by auto-combustion method. Rietveld refinement of X-ray diffraction data indicates a structural transition from $R 3 c$ to $R 3 c+P n 2_{1} a$ at $x=0.1$. Field emission scanning electron micrographs display a decrease in grain size with increase in $x$. The presence of dielectric anomalies around antiferromagnetic transition temperature implies the magnetoelectric coupling. Dielectric measurements showed decrease in magnetic ordering temperature with increasing $x$ in agreement with differential scanning calorimetry results. A significant increase in magnetization has been found with increasing $\mathrm{DyMnO}_{3}$ substitution. Magnetoimpedance spectroscopy reveals a significant change $(\sim 18 \%)$ in dielectric permittivity at $H=2 \mathrm{~T}$ for $x=0.2$. (C) 2015 AIP Publishing LLC. [http://dx.doi.org/10.1063/1.4916927]
\end{abstract}

\section{INTRODUCTION}

Multiferroic materials are drawing a lot of research interest due to their intriguing fundamental physics and potential applications in novel multifunctional devices. ${ }^{1-11}$ An essential feature of a multiferroic system is the presence of magnetoelectric (ME) coupling (not all multiferroics are magnetoelectric, e.g., $\mathrm{BaCoF}_{4}$ ) which permits a mutual control between ferroic order parameters (i.e., electric and magnetic ordering). ${ }^{2,11}$ Multiferroics are very rare in nature due to the chemical incompatibility and mutual exclusiveness of ferroelectric and ferromagnetic ordering in many oxides, more common among fluorides. ${ }^{3,11}$ In this context, $\mathrm{BiFeO}_{3}$ (BFO) with a distorted perovskite $\mathrm{ABO}_{3}$ structure is a proto-type multiferroic material owing to its high ferroelectric transition temperature $\left(T_{\mathrm{C}} \approx 830^{\circ} \mathrm{C}\right)$ and antiferromagnetic (AFM) Néel temperature $\left(T_{\mathrm{N}} \approx 370^{\circ} \mathrm{C}\right) .{ }^{4}$ The ferroelectricity in $\mathrm{BFO}$ is caused by the Bi 6s lone pair electrons, while spin of transition metal cation $\mathrm{Fe}^{3+}$ is responsible for G-type antiferromagnetic ordering with a long range spin cycloid with period of $620-640 \AA \AA^{4,5}$ Recent experimental investigations on $\mathrm{BFO}$ outline the major inherent issues, which should still be addressed: (a) synthesis of stoichiometric compound, (b) high leakage current and dielectric loss, and (c) weak magneto-electric coupling. ${ }^{1-5}$ The ferroelectric phase transition temperature is much higher than the AFM

\footnotetext{
a) Present Address: Institute of Physics, University of Silesia, Uniwersytecka 4, 40-007 Katowice, Poland. Electronic mail: satyanarayantripathy@ gmail.com. Tel.: +48-570469061.

b) Author to whom correspondence should be addressed. Electronic mail: dillip.pradhan79@gmail.com. Tel.: +91-661-2462729. Fax: +91-6612462999.
}

Néel temperature (i.e., Type-I multiferroic), and a weak magneto-electric coupling is observed. The origin of the problems mentioned above warrants further investigation. The preparation of stoichiometric $\mathrm{BFO}$ is a challenging problem because of its phase stabilization at very narrow temperature range and frequent presence of impurity phases, such as $\mathrm{Bi}_{2} \mathrm{Fe}_{4} \mathrm{O}_{9}, \mathrm{Bi}_{36} \mathrm{Fe}_{24} \mathrm{O}_{57}$, and $\mathrm{Bi}_{25} \mathrm{FeO}_{40}{ }^{6,7}$ Presence of these impurity phases results in high leakage current and poor/artificial multiferroic properties. ${ }^{4} \mathrm{BFO}$ also decomposes into starting materials under high temperature. ${ }^{6}$ Although, several synthesis techniques have been attempted to achieve phasepure material, only a few have been successful. On the other hand, BFO displays a very weak macroscopic magnetization (i.e., difficult to achieve saturated magnetic hysteresis loops) due to a spatially modulated spiral spin structure. ${ }^{2}$ The spiral spin structure can be suppressed or destroyed by suitable chemical substitution, fabrication of solid solutions, preparation of nano particles less than the size of the spin cycloid, application of high magnetic fields, and growing epitaxial thin film. ${ }^{7-13}$

BFO forms solid solutions with perovskite $\left(\mathrm{ABO}_{3}\right)$ and rare earth manganites $\left(\mathrm{REMnO}_{3}\right)$ over a wide compositional range and possess different structural symmetries which display intriguing multiferroic properties. ${ }^{2,9,12} \mathrm{REMnO}_{3}$ exhibits two distinct crystal structures depending on the ionic radius of the RE ions. Compounds containing RE element with large ionic radius $(\mathrm{RE}=\mathrm{La}, \mathrm{Pr}, \mathrm{Nd}, \mathrm{Sm}, \mathrm{Eu}, \mathrm{Gd}$, Dy, $\mathrm{Tb}$, and Dy) are stabilized in an orthorhombic "orthoferrite" structure (Pnma); and compounds containing RE element with a smaller ionic radius $(\mathrm{RE}=\mathrm{Ho}, \mathrm{Er}, \mathrm{Tm}, \mathrm{Yb}, \mathrm{Lu}, \mathrm{Y}, \mathrm{Sc}$ and In) possess hexagonal "hexaferrite" crystal structure with the $P 6_{3} \mathrm{~cm}$ space group. ${ }^{2,14-19}$ Both theoretical and 
experimental investigations on a series of rare-earth manganite with orthorhombic structure reveal that the origin of ferroelectricity is from the competing magnetic interactions, which produce long wavelength antiferromagnetic spin order and lattice modulations through magneto-elastic coupling. Furthermore, the coupling between lattice distortion and AFM ordering produces ferroelectricity (i.e., ferroelectric ordering directly related to magnetic order: Type-II multiferroics). As a result, it leads to strong magnetoelectric and giant magneto-dielectric effects. ${ }^{2,14-19} \mathrm{DyMnO}_{3}$ (DMO) shows $T_{\mathrm{N}}(\mathrm{Mn}) \approx 39 \mathrm{~K}$ corresponding to $\mathrm{AFM}$ ordering of Mn moments, $\mathrm{T}_{\text {lock }}\left(T_{\mathrm{C}}\right) \approx 18 \mathrm{~K}$ corresponding to development of ferroelectric ordering with appearance of spontaneous polarization and $T_{\mathrm{N}}(\mathrm{Dy}) \approx 10 \mathrm{~K}$ corresponding to AFM ordering of Dy ion moments. ${ }^{2,14-19}$ So it is expected that the synthesis of $(1-x) \mathrm{BiFeO}_{3}-x \mathrm{DyMnO}_{3}$ nanoceramic solid solutions could lower the magnetic ordering temperature of the parent BFO towards room temperature (RT). It has been demonstrated that $\mathrm{DyMnO}_{3}$ shows a giant magneto-dielectric effect with a change in dielectric constant up to $\approx 500 \%$ at $18 \mathrm{~K}$, which is an order of magnitude more than the previously studied rare-earth manganites, such as $\mathrm{TbMnO}_{3}{ }^{14-19}$ The Goodenough-Kanamori rule suggests that a strong ferromagnetic interaction is expected between the empty $\mathrm{Mn}^{3+}$ orbital and filled $\mathrm{Fe}^{3+}$ orbital. Asymptotically, a decrease in $T_{\mathrm{N}}$ toward room temperature could be accomplished by substitution of $\mathrm{Mn}^{3+}$ ions at the $\mathrm{Fe}^{3+}$-site of BFO. ${ }^{9,20}$ It has also been reported that dielectric losses, resistivity, and magnetic properties of $\mathrm{BFO}$ can be significantly enhanced by substitution of $\mathrm{Mn}^{3+}$ ion at the $\mathrm{Fe}^{3+}$ site. ${ }^{13}$ Chemical substitution of RE cation at the Bi-site of BFO has been established to be an effective approach to stabilize the perovskite phase and reduce the leakage current with improved multiferroic properties. ${ }^{12}$ The ionic radii of $\mathrm{Bi}^{3+}$ and $\mathrm{Dy}^{3+}$ are $1.17 \AA$ and $1.07 \AA$, respectively, for highspin eight-fold coordination, whereas the ionic radii of $\mathrm{Fe}^{3+}$ and $\mathrm{Mn}^{3+}$ are identical $(0.645 \AA)$ for high spin six-fold coordination. ${ }^{21} \mathrm{~A}$ further improvement in multiferroic properties in rare earth modified BFO nano particles has been observed due to the size effect. ${ }^{8,10}$ In the quest to have a multiferroic system with high ME coupling, nanoceramic solid solutions of Type-I $\left(\mathrm{BiFeO}_{3}\right)$ with Type-II $\left(\mathrm{DyMnO}_{3}\right)$ multiferroic systems have been synthesized and systematically investigated.

In this work, we have synthesized $(1-x) \mathrm{BiFeO}_{3}-x$ $\mathrm{DyMnO}_{3}(0.0 \leq x \leq 0.2)$ nanoceramic solid solutions and systematically investigated the structural, microstructural, vibrational, magnetic, temperature dependent dielectric, caloric, and magneto-dielectric properties. An attempt has been made to understand and correlate the magneto-electric coupling with structural properties.

\section{EXPERIMENTAL}

Solid solutions of $(1-x) \mathrm{BiFeO}_{3}-x \mathrm{DyMnO}_{3}$ (BFODMO) $(0.0 \leq x \leq 0.2)$ nanoceramics were prepared by an auto-combustion method using analytical grade chemicals of $\mathrm{Fe}\left(\mathrm{NO}_{3}\right)_{3} \quad 9 \mathrm{H}_{2} \mathrm{O}, \quad \mathrm{Bi}\left(\mathrm{NO}_{3}\right)_{3} \quad 5 \mathrm{H}_{2} \mathrm{O}, \quad \mathrm{Dy}_{2} \mathrm{O}_{3}$, and $\left(\mathrm{CH}_{3} \mathrm{COO}\right)_{2} \mathrm{Mn} 4 \mathrm{H}_{2} \mathrm{O}$ as starting materials (oxidizers) and urea as fuel. The reagents in stoichiometric amount were dissolved in water/nitric acid to prepare a homogenous gel. The fuel $(\mathrm{F})$ to oxidizer ratio $(\mathrm{O})$ was fixed to an optimized value $\mathrm{F} / \mathrm{O}=0.75$ for all compositions. ${ }^{22,23}$ Next the gel was transferred to the furnace, preheated at $400^{\circ} \mathrm{C}$ for $30 \mathrm{~min}$, depending upon requirements and conditions of the experiment. The gel suddenly gets auto-ignited to produce a voluminous ceramic oxide, known as combustion residue. The combustion residues were subsequently ground. Details of auto-combustion synthesis procedure are described in our previous work. ${ }^{22,23}$ For $x=0.0$, the combustion residue was calcined at an optimized temperature of $550^{\circ} \mathrm{C}$ for $3 \mathrm{~h}$ in air. The combustion residues of the samples $0.025 \leq x \leq 0.1$ were calcined at $700^{\circ} \mathrm{C}$ for $3 \mathrm{~h}$, while those for $x=0.15$ and $x=0.2$ were calcined at $750^{\circ} \mathrm{C}$ for $3 \mathrm{~h}$. The process of calcinations has been carried out at optimized conditions in terms of phase formation using X-ray diffraction (XRD) analysis. The calcined powders were pressed in form of the cylindrical pellets via a hydraulic press with pressure $8 \times 10^{7} \mathrm{~kg} \mathrm{~m}^{-2}$ using polyvinyl alcohol (PVA) as binder. The pellets of the samples $0.0 \leq x \leq 0.1$ and $0.15 \leq x \leq 0.2$ were sintered at optimized temperature of $750^{\circ} \mathrm{C}$ and $780^{\circ} \mathrm{C}$ for $6 \mathrm{~h}$, respectively. The phase formations of the compounds were examined by X-ray powder diffractometer (Philips Analytical-PW 3040) at room temperature. The XRD data were collected at very slow scan of $2^{\circ} / \mathrm{min}$ with a step size of $0.02^{\circ}$ in a wide range of Bragg's angle $2 \theta\left(20^{\circ} \leq 2 \theta \leq 80^{\circ}\right)$ using $\mathrm{Cu}-\mathrm{K} \alpha_{1}$ radiation $(\lambda=1.5405 \AA)$. Structural analysis of the solidsolution was carried out by Rietveld refinement technique using FULLPROF package. ${ }^{24} \mathrm{X}$-ray line-profile analysis (XLPA) method was used to estimate and analyze the crystallite size and strain in the samples using BREADTH software. $^{25}$ Field emission scanning electron microscope (FESEM) (SUPRA 35VP) was used to determine grain size, void, and uniformity of sample matrix. The energy dispersive X-ray analysis (EDX) was used to estimate the overall chemical composition of the samples. Transmission electron microscope (TEM) pattern and selected area diffraction pattern (SAED) were recorded using a TEM Techno $\mathrm{G}^{2} 30$ STwin. The Raman spectra were measured in the back scattering geometry using the $514.5 \mathrm{~nm}$ line of an Ar-ion laser in the frequency range $80-800 \mathrm{~cm}^{-1}$ using Renishaw microRaman spectrometer (model-INVIA). For electrical characterization, the pellets were polished and painted with silver electrodes and then dried at $150{ }^{\circ} \mathrm{C}$ for $3 \mathrm{~h}$ to remove moisture and organic solvents. The dielectric parameters (i.e., capacitance, loss tangent, impedance, phase angle) were measured in a wide frequency range (i.e., $100 \mathrm{~Hz}-1 \mathrm{MHz}$ ) using a computer-controlled impedance analyzer PSM-1735 in the temperature range $25-400^{\circ} \mathrm{C}$ with a laboratory designed sample holder. Calorimetric measurements on these systems were carried out by a Mettler-Toledo DSC apparatus equipped with liquid nitrogen cooling accessory and a HSS8 ceramic sensor (a heat flux sensor with 120 thermocouples). The magneto-dielectric measurements were performed using a vibrating sample magnetometer (VSM Lakeshore model $142 \mathrm{~A}$ ) and a HIOKI 3532-50 LCR Hi tester at room temperature. Magnetization hysteresis measurements (M-H hysteresis loop) were carried out at room temperature using a 
vibrating sample magnetometer (Lakshore 142AVSM) up to a maximum field of $\pm 2 \mathrm{~T}$ at ambient.

\section{RESULTS AND DISCUSSION}

\section{A. Structural and microstructural studies}

Figs. 1(a) and 1(b) display the room temperature XRD patterns of the solid solution of $(1-x)$ BFO- $x$ DMO, for $0.0 \leq x \leq 0.2$. Crystallographic analyses of the XRD data were carried out by Rietveld refinement technique using FULLPROF package. ${ }^{24}$ The peak shapes were refined using a pseudo-Voigt function, and background was modeled using a six-coefficient polynomial function. During the refinement process, zero correction, scale factor, background, unit cell parameters, atomic positions, thermal parameters, half width parameters $(U, V$, and $W)$, and background were varied. The occupancy of all the atomic sites was kept fixed during refinement process. The experimental data are labeled as symbol $(+)$, and simulated data are presented as solid lines shown in Fig. 1(a). The differences between observed and simulated patterns are denoted at the bottom of the plot. The rhombohedral crystal structure of pure $\mathrm{BiFeO}_{3}$ in $R 3 c$ space group was used for Rietveld refinement and the best agreement match (goodness of fit: $\chi^{2}=1.59$ ) is obtained between calculated and observed diffraction spectra. There are three ions $\left(\mathrm{Bi}^{3+} / \mathrm{Dy}^{3+}, \mathrm{Fe}^{3+} / \mathrm{Mn}^{3+}\right.$, and $\left.\mathrm{O}^{2-}\right)$ in the asymmetric unit of the rhombohedral perovskite structure. The cations $\mathrm{Bi}^{3+} / \mathrm{Dy}^{3+}$ and $\mathrm{Fe}^{3+} / \mathrm{Mn}^{3+}$ ions occupy the 6(a) Wyckoff site at $(0.0,0.0,0.3+\Delta \mathrm{z})$ and $(0.0,0.0,0.02+\Delta \mathrm{z})$, while $\mathrm{O}^{2-}$ anions are at the $18(\mathrm{~b})$ sites at $(0.23+\Delta \mathrm{x}, 0.34+\Delta \mathrm{y}$,
0.083). ${ }^{10}$ The various $\Delta \mathrm{s}$ ' represent the refinable parameters. The refined lattice parameters and volume of primitive unit cell of BFO are $a=5.5779$ (09) $\AA, c=13.8710$ (23) $\AA$, and $62.293 \AA^{3}$. These lattice parameters match with the reported values. ${ }^{4,7,10}$ It is observed that with increase in DMO content up to $x=0.075$, XRD patterns are similar to the parent $\mathrm{BiFeO}_{3}$ ( $R 3 c$ space group). This is evidenced by existence of characteristic superlattice (113) reflection around $\approx 38^{\circ}$ of rhombohedral $R 3 c$ phase, as shown in Fig. 1(b) in log scale. ${ }^{5}$ Therefore, the $R 3 c$ space group has been selected to refine the crystal structure up to $x=0.075$. The intensity magnitude of the superlattice peak (113) reflection decreases with increase in $x$. The (113) reflection arises from antiferrodistortive tilting of the octahedra, which gives rise to new oxygen planes resulting a low peak intensity. ${ }^{10}$ Splitting in the reflections, changes in peak position, peak intensity, and peak broadening in the diffraction patterns are noticed with increase in $x$. It was observed that for $x=0.2$, the XRD pattern is similar to $\mathrm{REFeO}_{3}(\mathrm{RE}=\mathrm{La}, \mathrm{Sm}, \mathrm{Gd}, \mathrm{Nd}, \mathrm{Dy})$ which is described by the PnmalPbnm space group. Note that Pnma/Pbnm structure is commonly observed in $\mathrm{ABO}_{3}$ crystals, rare earth ortho-ferrites, manganites and also observed as the high temperature crystal structure of $\mathrm{BiFeO}_{3} .{ }^{26}$ The polar $P n 2_{1} a$ space group is a subgroup of nonpolar Pnma space group. A superposition of two crystallographic phases, i.e., rhombohedral and orthorhombic phases is evidenced by (i) appearance of new reflection (111) around $\approx 25^{\circ}$; (ii) splitting of the reflection around $\approx 32^{\circ}$ into four peaks; and (iii) presence of superlattice (1 1 3 ) reflection, even beyond $x=0.075$ composition, as shown
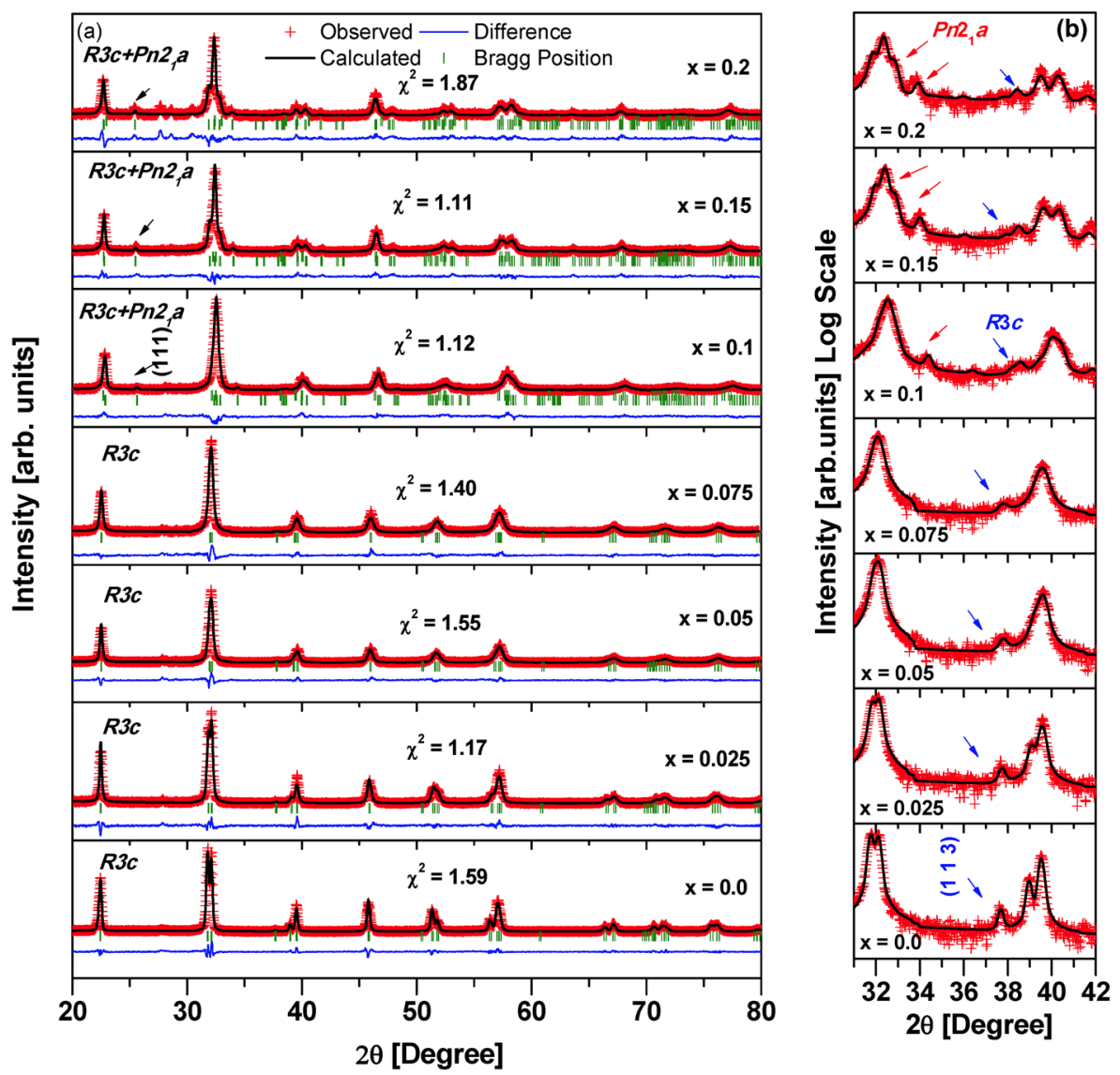

FIG. 1. Rietveld refinement result of room temperature $\mathrm{X}$-ray diffraction pattern of (1-x)BFO- $x$ DMO for $0.0 \leq x \leq 0.2$ in (a) linear scale, and (b) Log scale. 
in Fig. 1(b). Therefore, Rietveld fittings are undertaken using dual phase model $\left(R 3 c+P n 2_{1} a\right)$ for the composition range $0.1 \leq x \leq 0.2$ and yield a satisfactory fit. Similar type of dual phase space group is reported on A-site modified BFO; ${ }^{22-24}$ For $x=0.1$ composition, it is found that $85 \%$ is rhombohedral $R 3 c$ phase and the remaining $15 \%$ is orthorhombic $P n 2{ }_{1} a$ phase. The appearance of orthorhombic symmetry or change of crystal structure starting at $x=0.1$ (i.e., threshold composition) is the structural phase boundary of rhombohedral and orthorhombic phases. It was also found that $x=0.15$ contains $45.3 \%$ of $R 3 c$ and $54.7 \%$ of $P n 2{ }_{1} a$ phase and $x=0.2$ contains $25.8 \%$ of $R 3 c$ and $74.2 \%$ of $P n 2{ }_{1} a$ phase. Therefore for $x \geq 0.1$, coexistence of rhombohedral and orthorhombic phases with increase in orthorhombic phase fraction (i.e., decrease in $R 3 c$ phase fraction) with $x$ is evident. As for $x=0.20$, both the rhombohedral and orthorhombic phases are present; to predict the composition at which the system would enter the single phase Pn2 1 a (orthorhombic) symmetry state, we have used Lever rule. ${ }^{27}$ To examine the validity of this Lever rule, the phase fraction of both the phases has been plotted with different values of $x$ and is shown in Figure 2(a). From the graph, it is observed that the
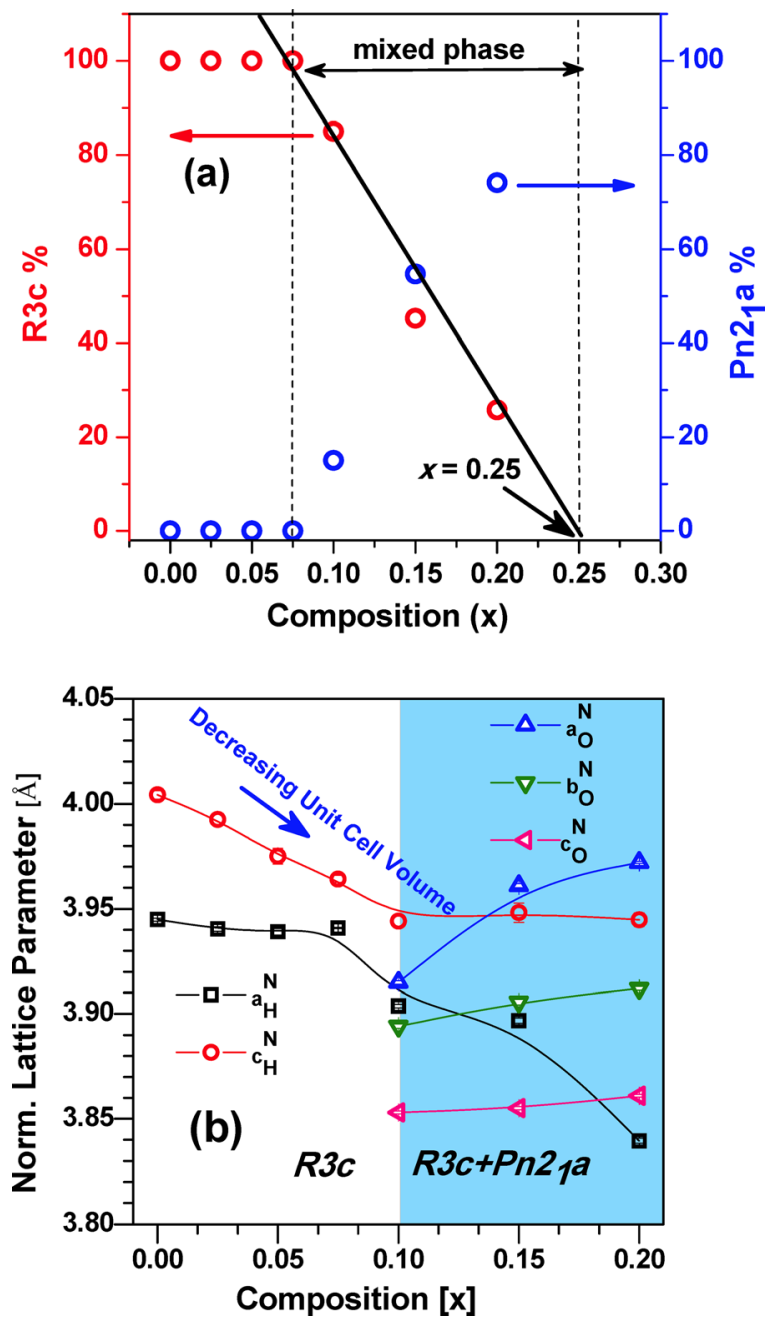

FIG. 2. (a) Variation of fraction of rhombohedral and orthorhombic phases with $x$ in $(1-x)$ BFO- $x$ DMO $(0.0 \leq x \leq 0.2)$ system. (b) Compositional dependence of normalized lattice parameters (i.e., $a_{\mathrm{H}}{ }^{\mathrm{N}}=a_{\mathrm{Hex}} / \sqrt{2}, c_{\mathrm{H}}{ }^{\mathrm{N}}=c_{\mathrm{Hex}} /$ $\sqrt{12}$ ) of $R 3 c$ phase and $P n 2_{1} a$ phase (i.e., $a_{\mathrm{O}}{ }^{\mathrm{N}}=b_{\mathrm{O}}{ }^{\mathrm{N}}=a_{\text {orth }} / \sqrt{ } 2, c_{\mathrm{O}}{ }^{\mathrm{N}}=c_{\text {orth }} / 2$ ). decrease in $R 3 c$ phase with composition $x$ follows a linear behavior. By extrapolating the decrease in $R 3 c$ phase to the composition axis (i.e., $x$-axis), it is noticed that for $x \geq 0.25$, the system enters to the single phase $P n 2{ }_{1} a$ (orthorhombic) symmetry state. Lee et al. using first principle calculation predicted the morphotropic phase boundary between rhombohedral and orthorhombic phases for $\mathrm{RE}=\mathrm{La}$ and $\mathrm{Sm}$ modified BFO (i.e., $\mathrm{Bi}_{1-\mathrm{x}} \mathrm{RE}_{\mathrm{x}} \mathrm{FeO}_{3}$ ) at $x=0.3$ and 0.14 , respectively. ${ }^{28}$ They have correctly reproduced the experimentally observed morphotropic phase boundary for rareearth substituted BFO multiferroics. They also reported this phase boundary to be unique for particular rare earth (RE) cation. However, in the present investigation, it can be inferred that for $0.1 \leq x \leq 0.25$, is the region of mixed phase and above $x=0.25$, the system enter to single symmetry phase of orthorhombic Pn $2_{1}$ a (orthorhombic) symmetry. The structural transformation is in our case could be due to many factors: (a) $\mathrm{Mn}^{3+}$ weakly destabilizes $R 3 c$ as compared to $\mathrm{Fe}^{3+}$ and $\mathrm{Dy}^{3+}$; (b) substitution of $\mathrm{Dy}^{3+}$ at Bi-site switches off the stereochemically active lone pair of $\mathrm{Bi}^{3+}$; (c) chemical pressure of $\mathrm{Dy}^{3+}$ is much smaller than for $\mathrm{Bi}^{3+}$ as the ionic radii of $\mathrm{Bi}^{3+}$ and $\mathrm{Dy}^{3+}$ are $1.17 \AA$ and $1.07 \AA$, respectively, for high-spin eight-fold coordination, whereas the ionic radii of $\mathrm{Fe}^{3+}$ and $\mathrm{Mn}^{3+}$ are identical (0.645 $\AA$ ) for high spin six-fold coordination. ${ }^{21}$ The Wyckoff notations and atomic positions for the coexisting $R 3 c$ and $P n 2_{1} a$ space groups are represented in Table S1 (See Supplementary Material $^{44}$ ). Beside this, no trace of impurity phases (i.e., $\mathrm{Bi}_{2} \mathrm{Fe}_{4} \mathrm{O}_{9}, \mathrm{Bi}_{25} \mathrm{FeO}_{39}$, etc.) was detected up to $x=0.15$. However, for $x=0.2$, presence of a very small impurity phase fractions could not be ruled out.

In order to probe the structural transition as a function of composition, the normalized lattice parameters (i.e., $\left.a_{\mathrm{H}}{ }^{\mathrm{N}}=a_{\mathrm{Hex}} / \sqrt{ } 2, c_{\mathrm{H}}{ }^{\mathrm{N}}=c_{\mathrm{Hex}} / \sqrt{ } 12\right)$ and (i.e., $a_{\mathrm{O}}{ }^{\mathrm{N}}=b_{\mathrm{O}}{ }^{\mathrm{N}}=a_{\text {orth }} / \sqrt{ } 2$, $\left.c_{\mathrm{O}}{ }^{\mathrm{N}}=c_{\mathrm{orth}} / 2\right)$ of $R 3 c$ and $P n 2_{1} a$, respectively, are shown in Fig. 2(b). A similar type of normalization technique has also been adopted by Selbach et al. ${ }^{5,10}$ This normalization technique brings the unit cell parameters of both the rhombohedral and orthorhombic space groups to a scaled magnitude for better comparison. With increase in composition, normalized lattice parameter of the $R 3 c$ phase decreases and approaches a similar magnitude for $x=0.1$. Beyond the structural phase boundary $x=0.1$, normalized lattice parameters of $R 3 c$ phase decrease sharply, whereas the $P n 2_{1} a$ phase increases with increase in composition. Hence, the orthorhombic phase becomes dominant at the expense of the rhombohedral phase. We observe that the structural transition is accompanied by sharp changes in unit cell parameters (i.e., discontinuous volume contraction abruptly from one constant value to another) at $x=0.1$. Below and above the threshold composition $x=0.1$, the primitive volume versus $x$ is nearly linear character. This implies the uniform distribution of dopants $\left(\mathrm{Dy}^{3+}\right.$ and $\left.\mathrm{Mn}^{3+}\right)$ throughout the lattice. ${ }^{29-31}$

The average crystallite size and lattice strain of $(1-x)$ BFO- $x$ DMO system for $0.0 \leq x \leq 0.2$ as a function of composition are shown in Fig. S1 (see Supplementary Material). In this context, we have adopted Fourier XLPA technique based on Double-Voigt method using program BREADTH. $^{25}$ Details of XLPA procedure are available in 
our previous work. ${ }^{22,23}$ For $x=0.0$, volume-weighted domain diameter $\left\langle D_{\mathrm{V}}\right\rangle$ ranges from 100 to $500 \AA$ with maximum around $260 \AA$; the distribution function, $\mathrm{P}_{\mathrm{v}}(\mathrm{L})$ becomes narrower with increasing $x$. This shows the uniform distribution of crystallite sizes. The average crystallite size varies in the range $200-300 \AA$ for our BFO-DMO system. In order to support the crystallite size calculations from X-ray diffraction data, we have carried out TEM measurement for $x=0.0$ and $x=0.1$, and the TEM micrograph and SAED pattern for $x=0.1$ are shown in Fig. S2 (see Supplementary Material). ${ }^{23}$ It is observed that the estimated average crystallize size is around $300 \AA$ from TEM characterization, which is in agreement with the XLPA calculation.

The DMO substitution effect on surface morphology and shape of the grains were observed by FESEM analyses. The FESEM micrographs of $(1-x)$ BFO- $x$ DMO system for $x=0.0, x=0.05, x=0.1$, and $x=0.2$ are displayed in Fig. 3 . Pure $\mathrm{BiFeO}_{3}(x=0.0)$ shows a non-uniform grain-size distribution with average size around $2 \mu \mathrm{m}$. With increase in $x$, average grain size decreases. The grains and grain boundaries are distinct for all compositions. As can be seen, sample with $x=0.1$ shows relatively some pores compared to other samples. The exact origin of the presence of more pores in $x=0.1$ sample is not quite clear. This could be due to the beginning of the mixed phases. The different temperature dynamics of the two phases could be responsible for the presence pores in $x=0.1$ sample. EDX of the $(1-x)$ BFO- $x$ DMO system confirms the presence of all the cationic elements in agreement within the stoichiometry within experimental error, as shown in Fig. S3 (see Supplementary Material).

\section{B. Raman spectroscopic studies}

$\mathrm{BiFeO}_{3}$ stabilizes in the rhombohedral crystal structure at ambient temperature and belongs to the space group $R 3 c{ }^{5}$ The group theoretical mode analysis predicts 13 modes, i.e., $\Gamma_{\text {opt }}=4 \mathrm{~A}_{1}+9 \mathrm{E}$, where $\mathrm{A}_{1}$ and $\mathrm{E}$ modes are both Raman and infrared active. ${ }^{32,33}$ Since the BFO-DMO has the same rhombohedral symmetry up to $x=0.075$ as in BFO, those

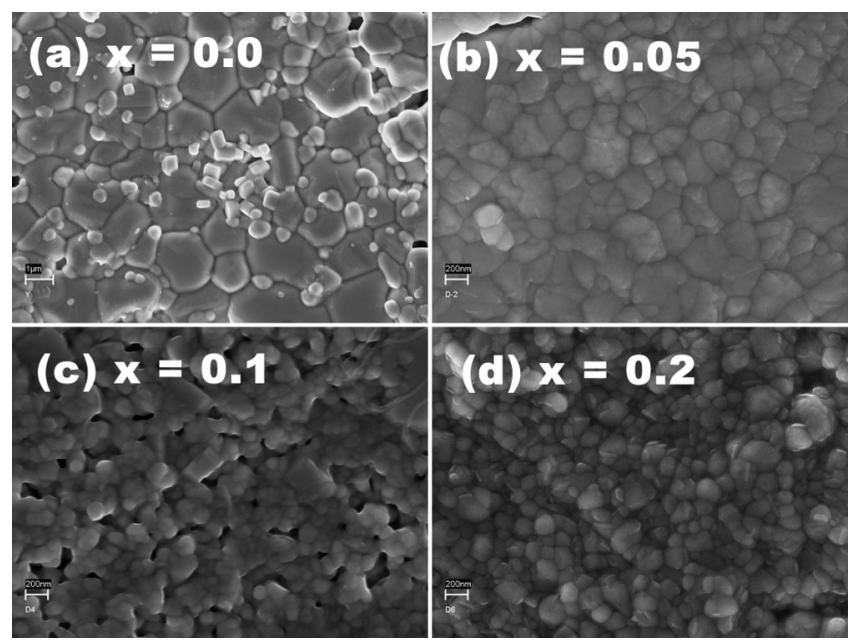

FIG. 3. FESEM micrographs of (a) $x=0.0$, (b) $x=0.05$, (c) $x=0.1$, and (d) $x=0.2$. predicted modes are also expected for these systems. Fig. 4(a) shows the Raman spectra of pure and DMO substituted BFO. For pure BFO, using PEAKFIT, ten modes could be identified in the frequency range $80-550 \mathrm{~cm}^{-1}$ (Fig. 4(b)). Lists of fitted phonon frequencies are tabulated in Table I and are assigned by comparing with those of BFO singlecrystals. ${ }^{32}$ One can notice that all modes are observed to be broadened as compared to those reported for single crystals. Broadening in Raman spectra is expected in polycrystalline samples since phonons scatter at grain boundaries. ${ }^{32}$ With increase in $x$, the peaks broadened further, and the intensities decreased. One can observe that the broadening of phonon modes becomes significant for $x=0.075$ and the spectral feature is too broad for $x=0.2$. For $x=0.075$ to $x=0.2$, only four Raman bands were found at 216, 290, 484, and $607 \mathrm{~cm}^{-1}$. For rhombohedral $x=0.075$ sample, expected signature of Raman features as in $x \leq 0.05$ is not observed. This could be due to broadening in vibration spectra and as a result, all the expected phonon modes are not resolved.
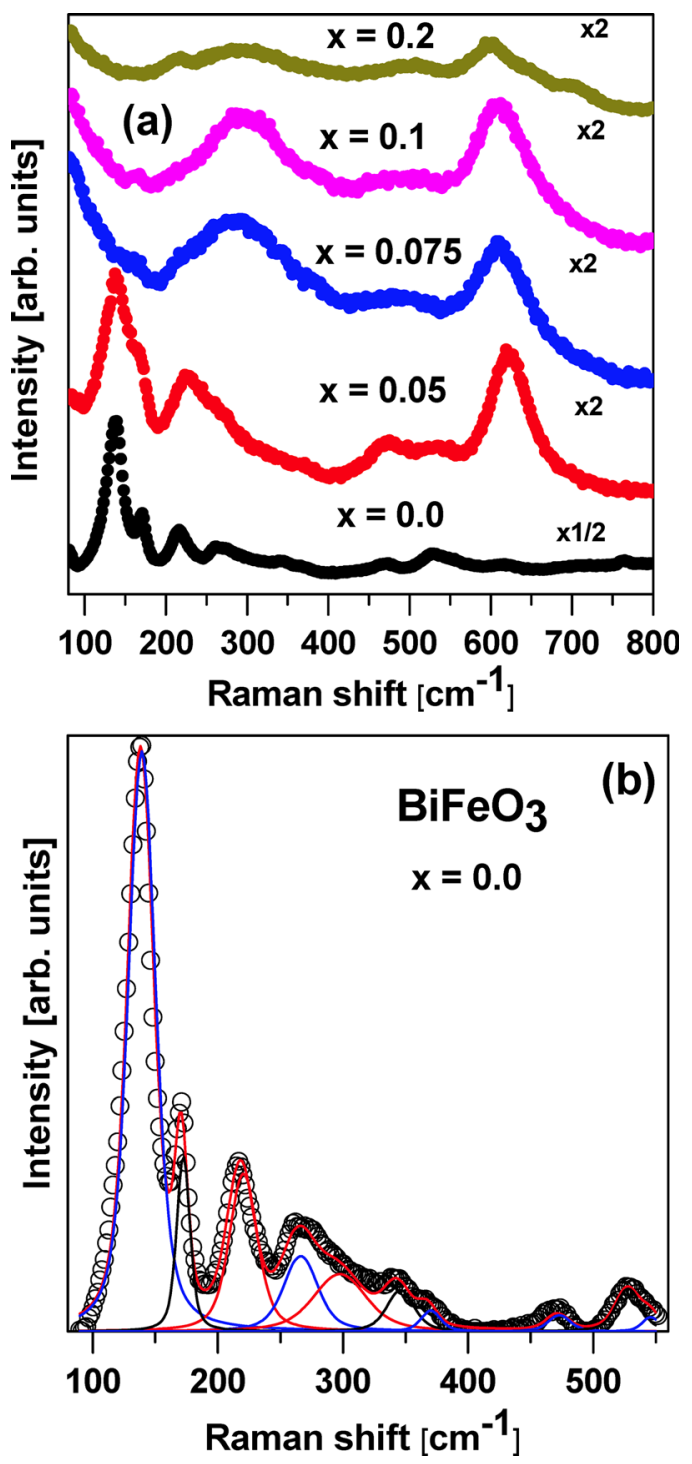

FIG. 4. (a) Raman spectra of $(1-x)$ BFO- $x$ DMO $(0.0 \leq x \leq 0.2)$ sample measured at ambient temperature and (b) Raman spectrum fitted to sum of 10 Lorentzian peaks for $x=0.0$ composition. Individual fitted peaks are also shown. 
TABLE I. Mode frequencies of the Raman bands in $\mathrm{BiFeO}_{3}$ and substituted $\mathrm{DyMnO}_{3}(x=0.2)$ system. The reported phonon frequencies for pure $\mathrm{BiFeO}_{3}$ single crystal and their assignments are also noted for comparison.

\begin{tabular}{lcccc}
\hline \hline $\begin{array}{l}(1-x) \mathrm{BiFeO}_{3}-x \mathrm{DyMnO}_{3} \text { present } \\
\text { work mode frequency }\left(\mathrm{cm}^{-1}\right)\end{array}$ & & \\
\cline { 1 - 2 }$x=0.0$ & $x=0.2$ & & Assignment & $\begin{array}{c}\mathrm{BiFeO}_{3} \text { mode } \\
\text { frequency }\left(\mathrm{cm}^{-1}\right)^{\mathrm{a}}\end{array}$ \\
\hline 138 & $\ldots$ & & E-TO & 145 \\
170 & $\ldots$ & & $\mathrm{A}_{1}-\mathrm{TO}$ & 168 \\
218 & 216 & & $\mathrm{~A}_{1}-\mathrm{TO}$ & 212 \\
263 & $\ldots$ & & $\mathrm{A}_{1}-\mathrm{LO}$ & 265 \\
294 & 290 & & E-TO & 295 \\
342 & $\ldots$ & & $\mathrm{A}_{1}-\mathrm{LO}$ & 350 \\
367 & $\ldots$ & & E-TO & 371 \\
471 & 484 & & E-TO & 473 \\
527 & $\ldots$ & & $\mathrm{A}_{1}-\mathrm{LO}$ & 523 \\
545 & 607 & E-TO & 553 \\
\hline \hline
\end{tabular}

${ }^{\mathrm{a}}$ c.f. Reference 32 .

Substitutional disorder often causes significant inhomogeneous broadening of Raman bands. With increase in $x$, the randomness of A-site occupancy by Bi/Dy and of the B-site by $\mathrm{Fe} / \mathrm{Mn}$ increases, and as a result, a distribution of bond lengths of ions often causes broadening. ${ }^{33}$ Although one can expect a signature of extra Raman-activated phonons due to the contribution of lower symmetry orthorhombic phase fraction $P n 2_{1} a$ for $x>0.075$, we could not identify such features. The difficulty in identifying such phonon modes is due to the significant inhomogeneous broadening of Raman bands and mixed selection rules of those modes. However, the four Raman bands of parent rhombohedral BFO continue to appear up to $x=0.2$, implying the existence of a rhombohedral phase fraction $R 3 c$ in agreement with our X-ray diffraction result.

\section{Magnetic properties}

Fig. 5 shows the magnetic hysteresis loops for $(1-x)$ BFO- $x$ DMO for $0.0 \leq x \leq 0.2$ solid solutions at RT. For $x=0.0$, the $M-H$ dependence shows a linear response without any spontaneous magnetization consistent with the antiferromagnetic nature of the sample. This supports the presence of canted G-type anti-ferromagnetism with spiral modulated spin structure. ${ }^{8}$ The magnetization increases sharply with increasing $x$ and reaches its maximum for $x=0.075$. The coercivity and remnant magnetization for $x=0.075$ are found to be $H_{\mathrm{c}}=200 \mathrm{mT}$ and $M_{\mathrm{r}}=0.05 \mathrm{emu}$ $\mathrm{gm}^{-1}$, respectively. However, with increase in composition, a discernable reduction in the coercivity (see Fig. 5(a)) is found after $x=0.1$. The magnetic hysteresis loops of the DMO modified BFO samples measured at RT indicate a typical ferromagnetic character. Néel theory suggests that such a large magnetic moment with reasonable hysteresis and the emergence of ferromagnetic-like behavior in small antiferromagnetic particles are attributed to incomplete spin compensation between the two sub-lattices. ${ }^{34}$ The observed enhancement in magnetization can be understood as follows: Either (a) with decrease in particle size, the surface-to-volume ratio rises significantly; each particle's average magnetization increases due to uncompensated spins at the surface; or (b) substitution of $\mathrm{Dy}^{3+}$ at A-site could lead to collapse of the modulated spin structure, and as a result, an improvement in magnetization is noticed. ${ }^{8}$ Since the magnetization is also affected by nearest neighbors, with increasing DMO concentration, the nearest neighbors of $\mathrm{Dy}^{3+}$ and $\mathrm{Fe}^{3+}$ have changed. This could be a reason for reduction of magnetization in BFO with higher $x$. Furthermore, $\mathrm{Dy}^{3+}$ ions are highly magnetic, which enhance magnetization and coercivity in the DMO-modified samples; yet the spiral modulated spin structure remains unchanged. For compositions below $x<0.1$ (i.e., in the $R 3 c$ phase of the DMO-modified BFO system), we have observed improved net magnetization. Fig. 5(b) shows that for $x \geq 0.1$, beyond the structural phase boundary composition, magnetization increases with field $H$, with very slim hysteresis. This implies a paramagnetic state in the $P n 2{ }_{1} a$ phase, which is evident from the fact that no hysteresis saturation was observed. In the structural transition from rhombohedral to orthorhombic in $\mathrm{BiFeO}_{3}$, the parent rhombohedral phase is responsible for spontaneous ferroelectricity; whereas the orthorhombic phase is responsible for appearance of weak ferromagnetism. ${ }^{30,34}$ Moreover, the X-ray characterization clearly demonstrates stoichiometric synthesis of the samples. The possibility of extrinsic contribution from the impurity phase $\mathrm{Bi}_{2} \mathrm{Fe}_{4} \mathrm{O}_{9}$ is negligible as
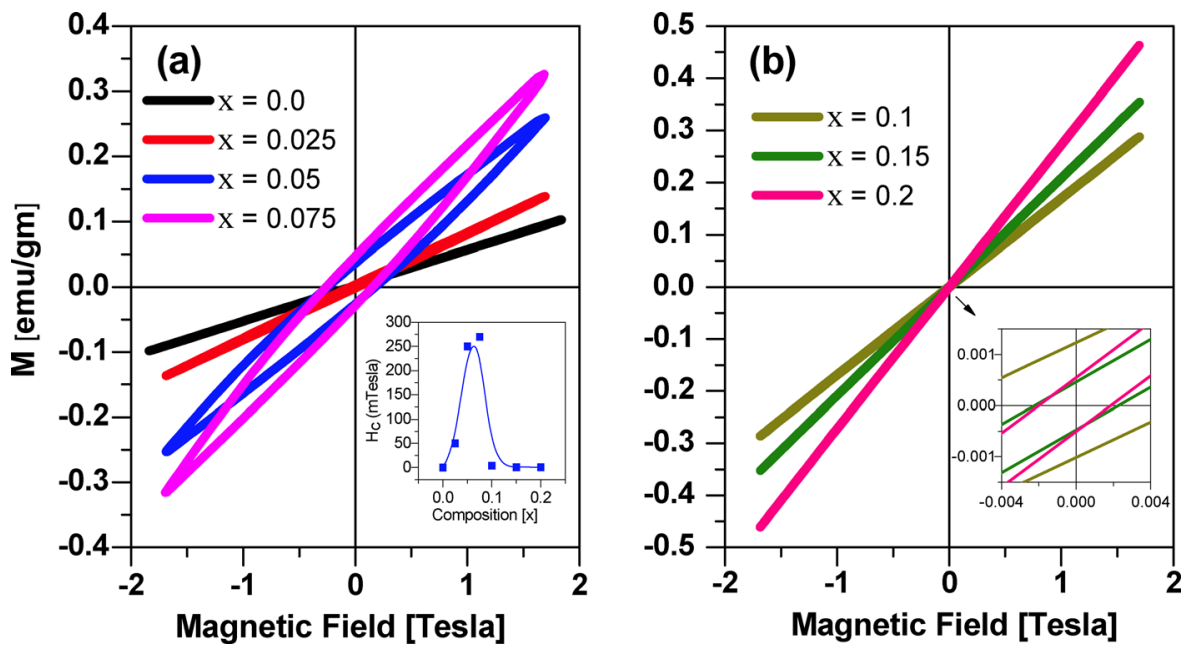

FIG. 5. (a) M-H hysteresis loops of $(1-x)$ BFO- $x$ DMO system at room temperature for (a) $0.0 \leq x \leq 0.075$ (inset shows compositional dependence of coercive field, $\mathrm{H}_{c}$ ). (b) $0.1 \leq x \leq 0.2$ (inset shows magnification of $\mathrm{M}-\mathrm{H}$ loop at lower fields). 
the magnetic ordering temperature of $\mathrm{Bi}_{2} \mathrm{Fe}_{4} \mathrm{O}_{9}$ is well below $\mathrm{RT}^{23,35}$ Therefore, we believe that the observation of the enhanced magnetization could be due to the dual effects of cycloid spin structure suppression and the structural $P n 2_{1} a$ instability due to the solid solution of BFO with DMO.

\section{Dielectric and calorimetric study}

To get insight into the presence of intrinsic magnetoelectric coupling in these multiferroic systems, the dielectric properties (i.e., dielectric permittivity and tangent loss) as a function of temperature and magnetic field are studied. ${ }^{14-19,35}$ Fig. 6(a) displays the dielectric permittivity as a function of temperature for the composition range $0.0 \leq x \leq 0.2$ at $1 \mathrm{MHz}$. To present unambiguous evidence of true magnetoelectric coupling at $T_{\mathrm{N}}$, here the dielectric data have been shown at sufficiently high frequency to demonstrate the effect of intrinsic bulk permittivity. ${ }^{36}$ It is clear from Fig. 6(a) [inset] that for $x=0.0$, the dielectric anomaly takes place around $365^{\circ} \mathrm{C}$. This anomaly corresponds to AFM transition of BFO. ${ }^{10}$ The appearance of this anomaly can be understood from the viewpoint of the Landau-
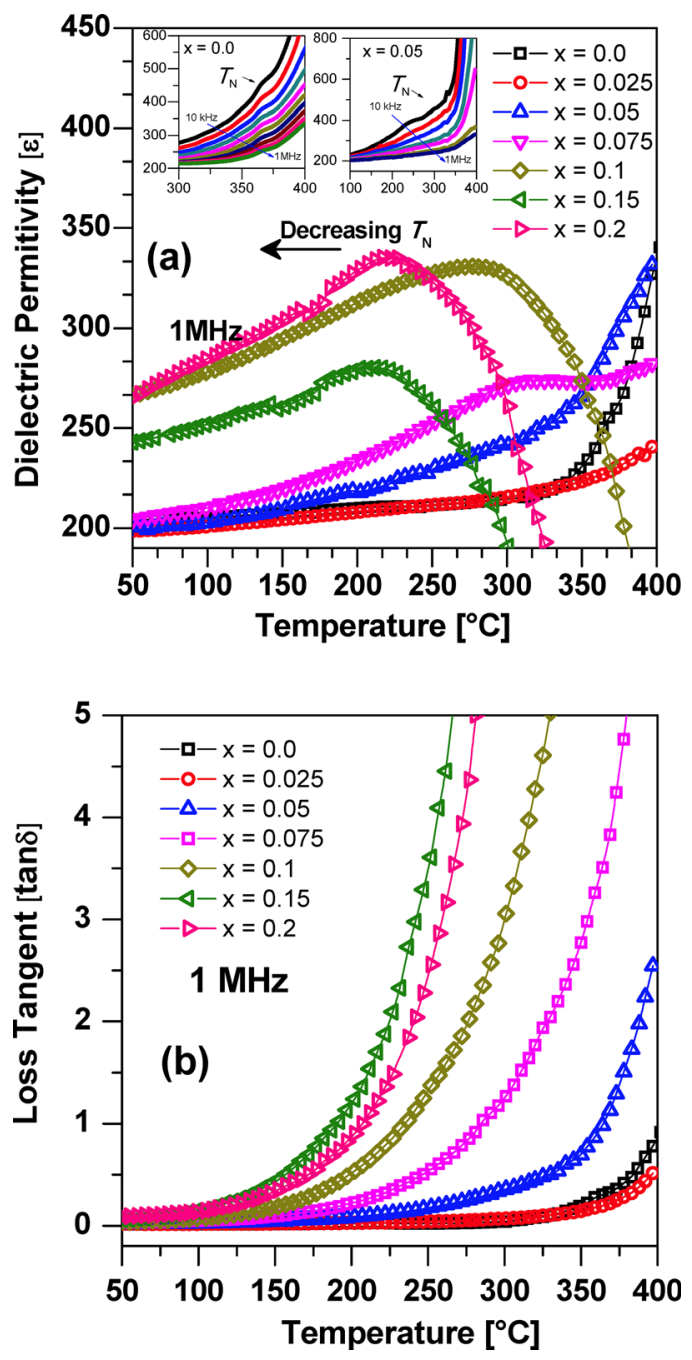

FIG. 6. Temperature dependent dielectric parameters of $(1-x)$ BFO- $x$ DMO system for $0.0 \leq x \leq 0.2$ : (a) $\varepsilon_{\mathrm{r}}$ vs. T at $1 \mathrm{MHz}$ (inset-temperature dependent $\varepsilon_{\mathrm{r}}$ at several frequencies between $10 \mathrm{kHz}$ and $1 \mathrm{MHz}$ for $x=0.0$ and $x=0.05)$ and (b) $\tan \delta$ vs. T at $1 \mathrm{MHz}$.
Devonshire theory and is attributed to the destruction of spatially modulated spin structure below the magnetic ordering temperature. ${ }^{35,37}$ Moreover, AFM- $T_{\mathrm{N}}$ decreases monotonically with increase in $x$, as evident from Fig. 6(a). Now two possibilities arise: First, $T_{\mathrm{N}}$ should increase with $x$ in $\mathrm{Bi}_{1-x} \mathrm{Dy}_{x} \mathrm{FeO}_{3}$. Here, $\mathrm{Dy}^{3+}$ destabilizes strongly the perovskite unit cell due to chemical pressure and there is a shrinking of unit cell volume and shortening of Fe-O bonds. This in turn translates to stronger antiferromagnetic superexchange coupling. ${ }^{4,5,10}$ Secondly, $T_{\mathrm{N}}$ should decrease in $\mathrm{BiFe}_{1-x} \mathrm{Mn}_{x} \mathrm{O}_{3}$ with increasing $x$ as $\mathrm{Mn}^{3+}$ is $d^{4}$ and $\mathrm{Fe}^{3+}$ is $d^{5}$. The Goodenough-Kanamori rule ${ }^{20}$ suggests that a strong antiferromagnetic interaction is probable between the $\mathrm{Fe}$ ions and a ferromagnetic interaction appear between the vacant $\mathrm{Mn}^{3+}$ orbital and filled $\mathrm{Fe}^{3+}$ orbital in perovskites. This breakdown the balance between the antiparallel sublattice magnetization of $\mathrm{Fe}^{3+}$, due to metal-ion substitution with a different valence. ${ }^{16}$ In the present study, it makes perfect sense that $T_{\mathrm{N}}$ decreases with increasing $\mathrm{DyMnO}_{3}$ content in $\mathrm{BiFeO}_{3}$, since the positive effect of $\mathrm{Dy}$ (on the $\mathrm{Bi}$

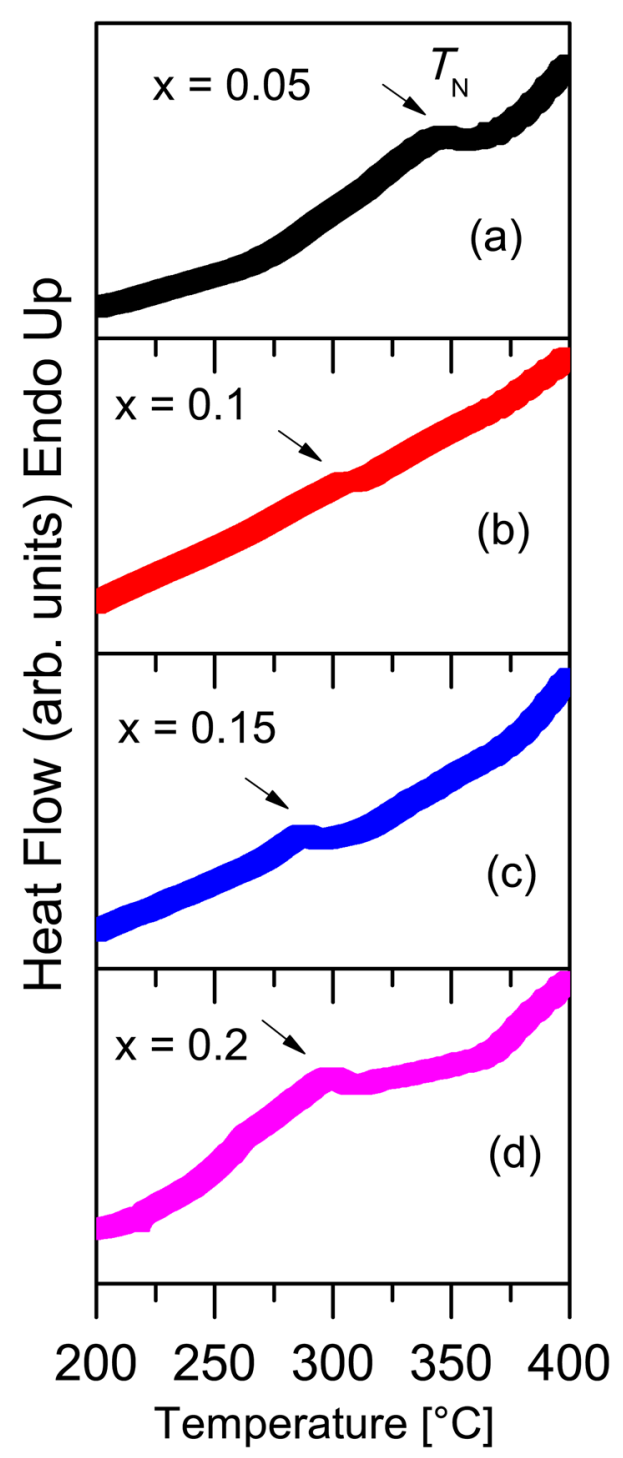

FIG. 7. DSC thermograms of $(1-x)$ BFO- $x$ DMO for (a) $x=0.05$, (b) $x=0.1$, (c) $x=0.15$, and (d) $x=0.2$. 

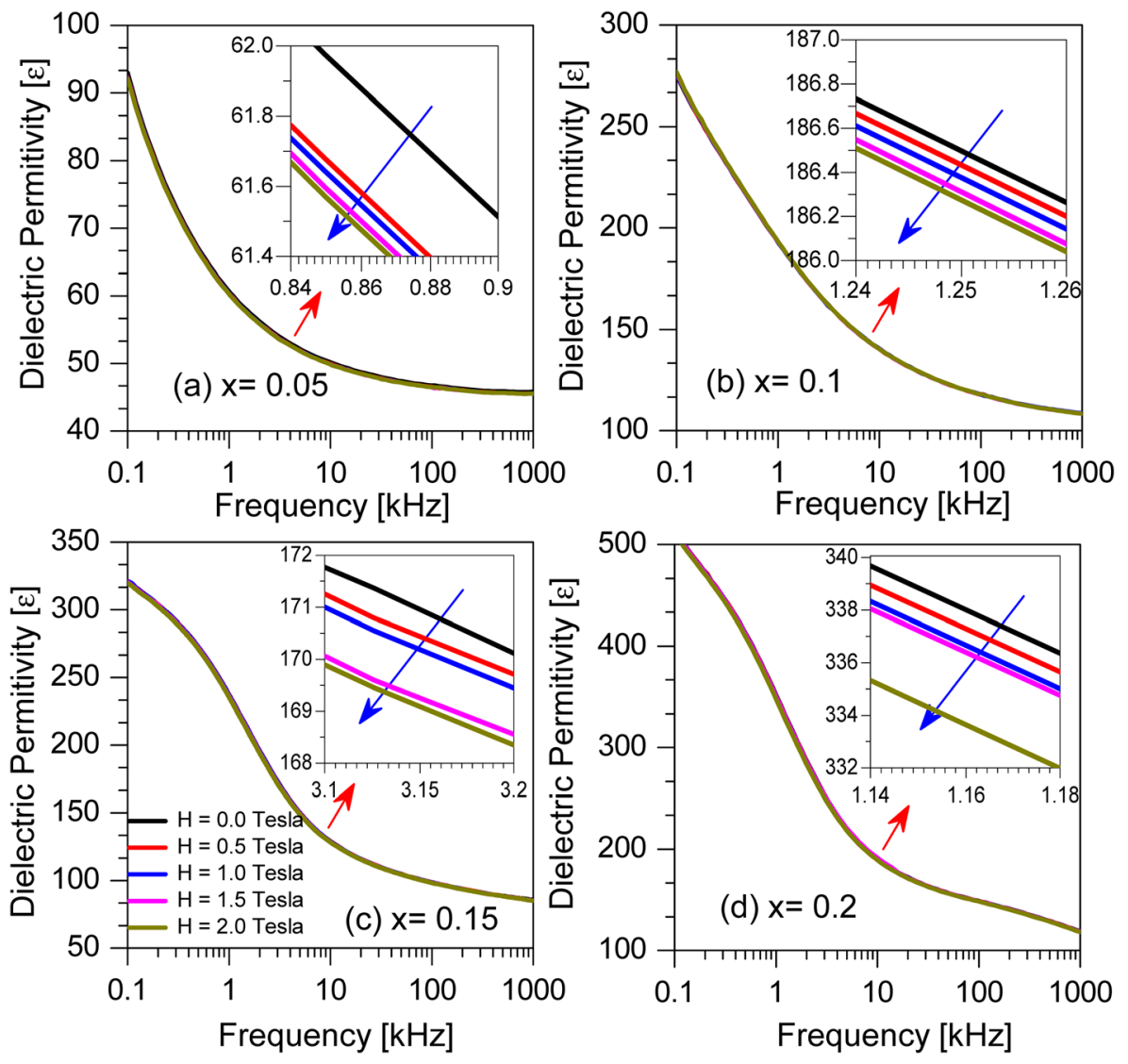

FIG. 8. Frequency dependent behavior of dielectric permittivity for (a) $x=0.05$, (b) $x=0.1$, (c) $x=0.15$, and (d) $x=0.2$ at different applied magnetic fields $(0 \leq \mathrm{H} \leq 2 \mathrm{~T}: \Delta \mathrm{H}=0.5 \mathrm{~T})$. The arrow indicates increasing magnetic field. sublattice) on $T_{\mathrm{N}}$ is much weaker than the negative effect of $\mathrm{Mn}$ (on the magnetic Fe sublattice). It is inferred that $\mathrm{Mn}^{3+}$ substitution at $\mathrm{Fe}^{3+}$ is predominantly responsible for driving the $\mathrm{AFM}-T_{\mathrm{N}}$ towards room temperature. Furthermore, the temperature dependent loss tangent increases with increase in temperature and composition as well, as shown in Fig. 6(b), due to the increase in the d.c. conductivity. The observed high dielectric loss at very low frequency could be due to the manifestation of space charge polarization, interfacial polarization (Maxwell Wagner effect), or presence of traps and defects. In order to understand the dielectric anomaly at $T_{\mathrm{N}}$, we carried out calorimetric measurements for $x=0.05, x=0.1, x=0.15$, and $x=0.2$ samples, as shown in Fig. 7. For all compositions $x$, the DSC is characterized by an endothermic peak near the AFM transition temperature. The DSC confirms that the endothermic peak temperature is nearly same as observed in the dielectric study for these materials within experimental uncertainty. It is observed that $T_{\mathrm{N}}$ decreases with increase in $x$. Therefore, we conclude that the anomalies observed in temperature dependent dielectric studies are due to the magnetodielectric coupling. The magnetic field dependent behavior of dielectric permittivity is discussed in Sec. III E.

\section{E. Magnetoelectric coupling}

For the better understanding of the ME coupling, we measured magneto-dielectric and magneto-impedance (MI) properties as a function of frequency at RT. The presence of ME coupling is evident from the dielectric measurements as follows: ${ }^{38,39}$ (a) an anomaly around $T_{\mathrm{N}}$ in the dielectric permittivity; and (b) a change in dielectric permittivity by the application of magnetic field. The presence of dielectric anomaly around the $\mathrm{T}_{\mathrm{N}}$ in the temperature dependent dielectric permittivity (Fig. 6) at high frequency (i.e., $1 \mathrm{MHz}$ ) clearly indicates the existence of magnetoelectric coupling, which is further confirmed by the decreasing of dielectric permittivity by the application of magnetic fields (Fig. 8). The exact mechanism of the origin of the ME coupling is not clear, however, it could be possible due to the magnetostriction and piezostriction. The Lorentz force can suppress the space charge polarization at very low frequency. In generally, the interfacial space charge polarizations are active up to $\sim 200-300 \mathrm{~Hz}$ range. ${ }^{40}$ Our measurements were carried out up to $\mathrm{MHz}$ range. We believe that the observed $\mathrm{ME}$ effects are intrinsic in nature. In general, a strong MC effect in a material is an indication of a strong MD coupling. However, some heterogeneous systems (involving interfacial or grain boundary layers or superlattices) and systems with the mismatch of work function at the electrode-dielectric interfaces can cause a strong MC effect, not related to any $\mathrm{ME}$ and MD coupling. These extrinsic effects can be ruled out by MI measurements. Therefore, we have analyzed the magneto-impedance spectroscopy. Fig. 9 shows the Im $\left(-Z^{\prime \prime}\right)$ vs. Re $\left(Z^{\prime}\right)$ part of the impedance (i.e., Nyquist plot) at RT for $x=0.05, x=0.1, x=0.15$, and $x=0.2$ at different applied magnetic fields $(0 \leq H \leq 2 \mathrm{~T})$. The impedance spectra are characterized by appearance of two overlapping semicircular arcs with the center below the real axis implies CPE (constant phase element). The CPE index in pure BFO is $\mathrm{n}=0.95 .^{38,41-43}$ The high frequency semicircle corresponds to bulk contribution, whereas the low frequency arc is 

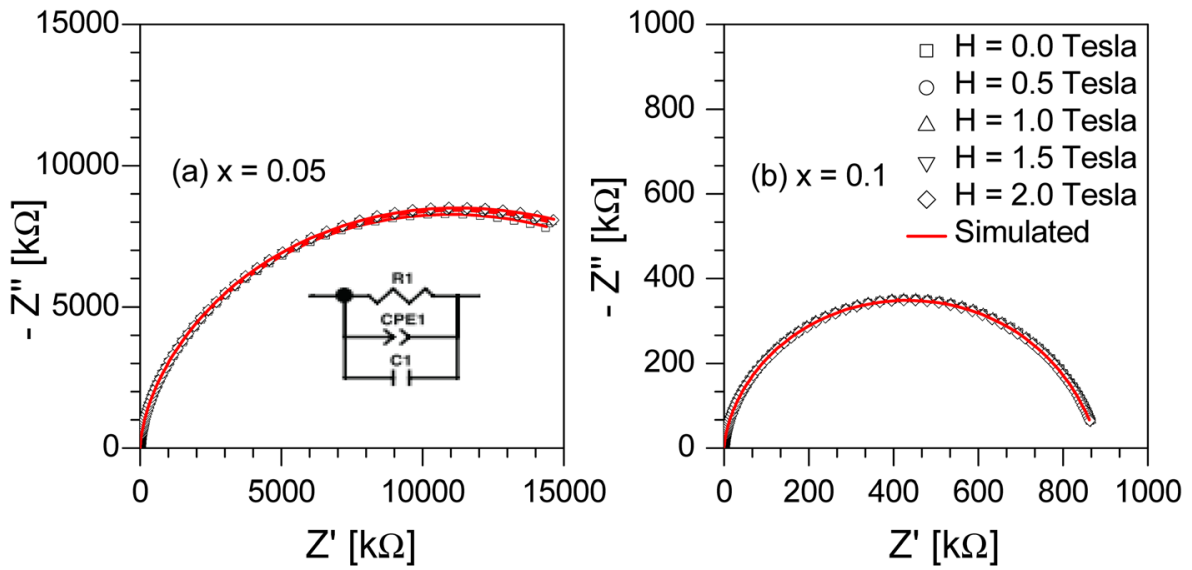

FIG. 9. Magnetic field dependent complex impedance plot (open symbol), fitted data (solid line) of $(1-x)$ BFO- $x$ DMO for (a) $x=0.05$, (b) $x=0.1$, (c)
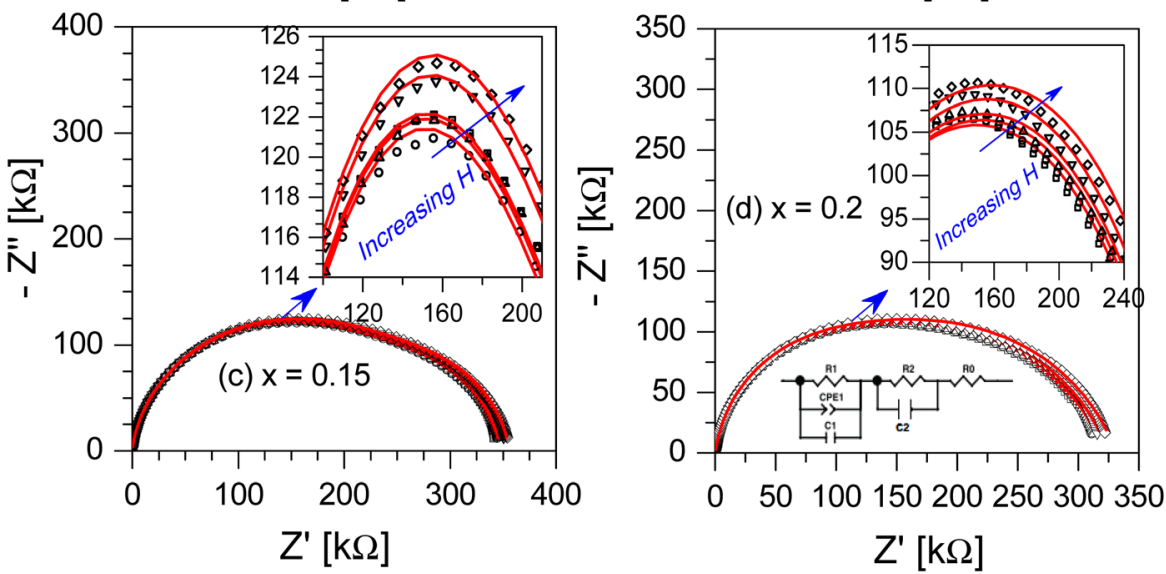
$x=0.15$, and (d) $x=0.2$ at room temperature. Inset shows the equivalent circuit to fit the impedance data.

TABLE II. Bulk resistance obtained from fitting of magnetic field dependent complex impedance plot at RT for all values of $x$.

\begin{tabular}{|c|c|c|c|c|c|c|c|}
\hline \multicolumn{8}{|c|}{ Bulk resistance in $\mathrm{Ohm}$} \\
\hline Field (T) & $\begin{array}{c}x=0.0 \\
10^{6}\end{array}$ & $\begin{array}{c}x=0.025 \\
10^{7}\end{array}$ & $\begin{array}{c}x=0.05 \\
10^{7}\end{array}$ & $\begin{array}{c}x=0.075 \\
10^{6}\end{array}$ & $\begin{array}{c}x=0.1 \\
10^{5}\end{array}$ & $\begin{array}{c}x=0.15 \\
10^{5}\end{array}$ & $\begin{array}{c}x=0.2 \\
10^{5}\end{array}$ \\
\hline 0.0 & 8.6176 & 1.844 & 2.553 & 4.456 & 7.659 & 2.739 & 2.404 \\
\hline 0.5 & 9.3114 & 1.857 & 2.592 & 4.48 & 7.721 & 2.741 & 2.388 \\
\hline 1.0 & 13.469 & 1.885 & 2.605 & 4.505 & 7.783 & 2.755 & 2.403 \\
\hline 1.5 & 14.609 & 1.879 & 2.613 & 4.513 & 7.708 & 2.802 & 2.452 \\
\hline 2.0 & 15.589 & 1.881 & 2.621 & 4.518 & 7.713 & 2.819 & 2.472 \\
\hline
\end{tabular}

attributed to grain boundary response. We have fitted the magnetic field dependent Nyquist plot using the equivalent circuit as shown in Fig. 9 and found a close agreement between the experimental and the simulated data for all values of $x$. The justification of proposed equivalent circuit is

TABLE III. Bulk capacitance obtained from fitting of magnetic field dependent complex impedance plot at RT for all values of $x$.

Bulk capacitance $\left[10^{-11}\right]$ in $\mathrm{F}$

\begin{tabular}{lccccccc} 
Field (T) & $x=0.0$ & $x=0.025$ & $x=0.05$ & $x=0.075$ & $x=0.1$ & $x=0.15$ & $x=0.2$ \\
\hline 0.0 & 4.174 & 2.393 & 2.254 & 2.426 & 5.21 & 3.727 & 5.249 \\
0.5 & 4.145 & 2.385 & 2.243 & 2.419 & 5.19 & 3.71 & 4.37 \\
1.0 & 4.152 & 2.385 & 2.243 & 2.403 & 5.257 & 3.707 & 4.38 \\
1.5 & 4.152 & 2.383 & 2.242 & 2.402 & 5.199 & 3.705 & 4.516 \\
2.0 & 4.152 & 2.383 & 2.242 & 2.402 & 5.193 & 3.701 & 4.292 \\
\hline \hline
\end{tabular}

reported elsewhere. ${ }^{9}$ It was observed that with increase in magnetic field the diameter of semicircle increases representing enhancement in bulk resistance (see Table II). Moreover, it is important that the bulk capacitance decreases with increase in magnetic field as represented in Table III for all $x$. This observation implied the existence of direct magnetoelectric coupling. The magnitude of maximum mgnetocapacitance $M C \%=\frac{\varepsilon(H, T)-\varepsilon(0, T)}{\varepsilon(0, T)}$ increased up to $-18 \%$ at $H=2 \mathrm{~T}$ for $x=0.2$. Since our sample is very resistive at RT and the measurement frequency range is not sufficiently high (i.e., more than $1 \mathrm{MHz}$ ), we did not notice any cross-over from capacitive to inductive behavior in the frequency dependent dielectric study. As it is well known the imaginary part of the impedance usually goes from negative (capacitive) to positive (inductive) values at sufficiently high frequency. Therefore, we have not selected the inductive parameter in our equivalent circuit model.

\section{SUMMARY AND CONCLUSIONS}

In conclusion, we successfully synthesized solid solutions of $(1-x) \mathrm{BiFeO}_{3}-x \mathrm{DyMnO}_{3}$, for $0.0 \leq x \leq 0.2$, using an autocombustion method. $\mathrm{DyMnO}_{3}$ substitution induced a structural phase transition from $R 3 c$ to $R 3 c+P n 2_{1} a$ at $x=0.1$. A decrease in grain size on increasing composition was observed from FESEM. The presence of an anomaly around the magnetic phase transition in dielectric permittivity as a function of temperature and the observation of magneto-capacitance and magneto-impedance effect at RT indicate the existence of magneto-electric coupling in DMO modified BFO samples. Dielectric studies corroborated the calorimetric results and 
showed that the magnetic ordering temperature decreases with increasing composition. Improved magnetization properties were observed at RT with increasing $x$. The method of magneto-impedance spectroscopy unambiguously demonstrated the influence of magnetic field on intrinsic bulk permittivity. Therefore, solid solutions of BFO with DMO may play a key role in improvement of the multiferroic properties of this important material.

\section{ACKNOWLEDGMENTS}

This work is partially supported by DST fast track Project No. SR/FTP/PS-16/2009. Dhiren K. Pradhan acknowledges IFN (NSF Grant No. EPS-01002410) for fellowship. The work at UPR was supported by National Science Foundation (NSF DMR 1410869) and Institute for Functional Nanomaterials (IFN). We also acknowledge Dr. Rainer Schmidt (Universidad Complutense de Madrid, GFMC, Dpto. Física Aplicada III, Facultad de Ciencias Físicas, Madrid, Spain) for fruitful suggestion on magnetoimpedance spectroscopy.

${ }^{1}$ M. Fiebig, J. Phys. D: Appl. Phys. 38, R123 (2005).

${ }^{2}$ K. F. Wang, J. M. Liu, and Z. F. Ren, Adv. Phys. 58, 321 (2009).

${ }^{3}$ N. Hill, J. Phys. Chem. B 104, 6694 (2000).

${ }^{4}$ G. Catalan and J. F. Scott, Adv. Mater. 21, 2463 (2009).

${ }^{5}$ S. M. Selbach, T. Tybell, M. Einarsrud, and T. Grande, Adv. Mater. 20, 3692 (2008).

${ }^{6}$ M. Valant, A. Axelsson, and N. Alford, Chem. Mater. 19, 5431 (2007).

${ }^{7}$ R. Palai, R. S. Katiyar, H. Schmid, P. Tissot, S. J. Clark, J. Robertson, S. A. T. Redfern, G. Catalan, and J. F. Scott, Phys. Rev. B 77, 014110 (2008).

${ }^{8}$ T. J. Park, G. C. Papaefthymiou, A. J. Viescas, A. R. Moodenbaugh, and S. S. Wong, Nano Lett. 7, 766 (2007).

${ }^{9}$ D. K. Pradhan, R. N. P. Choudhary, C. Rinaldi, and R. S. Katiyar, J. Appl. Phys. 106, 024102 (2009).

${ }^{10}$ S. M. Selbach, T. Tybell, M. A. Einarsrud, and T. Grande, Chem. Mater. 19, 6478 (2007).

${ }^{11}$ J. F. Scott and R. Blinc, J. Phys.: Condens. Matter 23, 113202 (2011).

${ }^{12}$ C. Yang, D. Kan, I. Takeuchi, V. Nagarajan, and J. Seidel, Phys. Chem. Chem. Phys. 14, 15953 (2012).

${ }^{13}$ D. Do, J. Kim, and S. Kim, J. Am. Ceram. Soc. 94, 2792 (2011).

${ }^{14}$ J.-H. Lee, P. Murugavel, D. Lee, T. W. Noh, Y. Jo, M.-H. Jung, K. H. Jang, and J.-G. Park, Appl. Phys. Lett. 90, 012903 (2007).

${ }^{15}$ T. Kimura, T. Goto, H. Shintani, K. Ishizaka, T. Arima, and Y. Tokura, Nature 426, 55 (2003).

${ }^{16}$ T. Kimura, S. Ishihara, H. Shintani, T. Arima, K. T. Takahashi, K. Ishizaka, and Y. Tokura, Phys. Rev. B 68, 060403 (R) (2003).
${ }^{17}$ T. Kimura, G. Lawes, T. Goto, Y. Tokura, and A. P. Ramirez, Phys. Rev. B 71, 224425 (2005).

${ }^{18}$ T. Goto, T. Kimura, G. Lawes, A. P. Ramirez, and Y. Tokura, Phys. Rev. Lett. 92, 257201 (2004).

${ }^{19}$ H. L. Yakel, W. D. Koehler, E. F. Bertaut, and F. Forrat, Acta Crystallogr. 16, 957 (1963).

${ }^{20}$ C.-H. Yang, T. Y. Koo, and Y. H. Jeong, Solid State Commun. 134, 299 (2005).

${ }^{21}$ R. D. Shannon and C. T. Prewitt, Acta Crystallogr., Sect. B: Struct. Crystallogr. Cryst. Chem. 25, 925 (1969).

${ }^{22}$ S. N. Tripathy, B. G. Mishra, M. M. Shirolkar, S. Sen, S. R. Das, D. B. Janes, and D. K. Pradhan, Mater. Chem. Phys. 141, 423 (2013).

${ }^{23}$ S. N. Tripathy, K. K. Mishra, S. Sen, B. G. Mishra, D. K. Pradhan, R. Palai, and D. K. Pradhan, J. Appl. Phys. 114, 144104 (2013).

${ }^{24}$ J. Rodriguez-Carvajal, Physica B 192, 55 (1993).

${ }^{25}$ D. Balzar, J. Appl. Crystallogr. 28, 244 (1995).

${ }^{26}$ R. Haumount et al., Phys. Rev. B 79, 184110 (2009).

${ }^{27}$ W. Cao and L. E. Cross, Phys. Rev. B 47, 4825 (1993).

${ }^{28}$ J. H. Lee, M. A. Oak, H. J. Choi, J. Y. Son, and H. M. Jang, J. Mater. Chem. 22, 1667 (2012).

${ }^{29}$ N. Li, J. Wu, Y. Jiang, Z. Xie, L. Zheng, and Z. Ye, J. Appl. Phys. 113, 054102 (2013).

${ }^{30}$ V. A. Khomchenko et al., J. Appl. Phys. 103, 024105 (2008).

${ }^{31}$ V. A. Khomchenko et al., J. Appl. Phys. 108, 074109 (2010).

${ }^{32}$ R. Palai, H. Schmid, J. F. Scott, and R. S. Katiyar, Phys. Rev. B 81, 064110 (2010).

${ }^{33}$ K. K. Mishra, V. Sivasubramanian, R. M. Sarguna, T. R. Ravindran, and A. K. Arora, J. Solid State Chem. 184, 2381 (2011).

${ }^{34}$ F. Z. Qian, J. S. Jiang, S. Z. Guo, D. M. Jiang, and W. G. Zhang, J. Appl. Phys. 106, 084312 (2009).

${ }^{35}$ G. L. Yuan and S. Or, J. Appl. Phys. 100, 024109 (2006).

${ }^{36}$ G. Catalan, Appl. Phys. Lett. 88, 102902 (2006).

${ }^{37}$ L. Benguigui, Solid State Commun. 11, 825 (1972).

${ }^{38}$ R. Schmidt et al., Phys. Rev. B 86, 035113 (2012).

${ }^{39}$ V. R. Palkar, D. C. Kundaliya, S. C. Malik, and S. Bhattacharya, Phys. Rev. B 69, 212102 (2004).

${ }^{40}$ K. C. Kao, Dielectric Phenomena in Solids (Elsevier Academic Press, 2004), p. 99.

${ }^{41}$ F. D. Morrison, D. J. Jung, and J. F. Scott, J. Appl. Phys. 101, 094112 (2007).

${ }^{42}$ R. Mart'inez, A. Kumar, R. Palai, J. F. Scott, and R. S. Katiyar, J. Phys. D: Appl. Phys. 44, 105302 (2011).

${ }^{43}$ R. Schmidt, W. Eerenstein, T. Winiecki, F. D. Morrison, and P. A. Midgley, Phys. Rev. B 75, 245111 (2007).

${ }^{44}$ See supplementary material at http://dx.doi.org/10.1063/1.4916927 for [I. (a) Volume distribution function $\mathrm{P}_{\mathrm{V}}(\mathrm{L})$ vs. crystallite size, (b) crystallite size and RMS strain estimated from Double-Voigt method as a function of composition of $(1-x) \mathrm{BiFeO}_{3}-x \mathrm{DyMnO}_{3}$ system. II. TEM micrograph for $x=0.1$ along with SAED pattern with inset. III. EDX spectra of $(1-x)$ $\mathrm{BiFeO}_{3}-x \mathrm{DyMnO}_{3}$ system for $x=0.00, x=0.5, x=0.1$, and $x=0.2$. IV. Wyckoff notation and atomic positions of $(1-x)$ BFO- $x$ DMO for $0.0 \leq x \leq 0.2$ used in Rietveld refinement. The standard deviations are in parenthesis for $x=0.2$ ] 